\title{
Microstructured head-up display screen for automotive applications
}

\author{
M. Kivanc Hedili*a ${ }^{\text {, Mark O. Freeman }}{ }^{\mathrm{b}}$, Hakan Urey ${ }^{\mathrm{a}}$ \\ ${ }^{a}$ Optical Microsystems Laboratory, Koç University, Rumelifeneri Yolu, 34450 Sarıer, İstanbul,

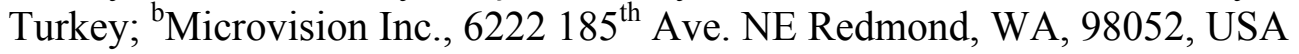

\begin{abstract}
A novel see-through screen is developed for automobiles which reduces the size of the head-up display (HUD) unit considerably. The screen is illuminated by a laser scanning pico-projector and a real image is formed on the screen. The screen has thousands of hexagonally packed microlenses that are partially reflective and embedded in an index matched medium which provides very good see-through capability. Light reflected from the microlenses expand and form a hexagon shaped viewing window. This system is called a direct projection HUD system as the pico projector projects directly onto the screen and forms a real image on it. The system is very compact and does not require any space under the dashboard, which saves on space for the car manufacturers, or allows it to be used immediately as an aftermarket HUD installed in any car.
\end{abstract}

Keywords: micro-optics, head-up displays, displays, transparent screens

\section{INTRODUCTION}

Head-up display (HUD) systems are becoming a part of the standard equipment in vehicles. It is shown that HUDs significantly reduce the response time of the drivers compared to head-down displays (HDD) by keeping the driver's eyes on the road ahead ${ }^{1}$. Virtual image HUDs, that is HUDs where the displayed image appears to be floating in space over the front of the car, are the most common design found in cars today. They have the advantage that the driver can view the road scene and the HUD information without having to refocus her eyes. However, the virtual image comes at a cost in the volume needed to house the HUD unit. Virtual image HUDs are bulky due to the imaging and relay optics required to create a virtual image outside the windshield ${ }^{2}$. Vehicles have to be designed specifically to accommodate the virtual image HUD unit, which occupies a large space (1-2 liters for HUDs in cars today) under the dashboard.

The invention of mobile, hand-held projectors, as illustrated in Fig.1, has opened a new path in compact HUD system design for cars. The comparison and technical description of virtual-image HUDs and direct-projection HUDs was introduced in ref. [2]. Unlike virtual image HUDs, with a direct-projection HUD, the image plane is on the combiner itself; it is not a virtual image outside the windshield. Since the content is projected directly onto the screen forming a real image, it is called a direct projection HUD. The driver's eyes remain on the road scene ahead, but shift in focus to read the HUD information. With this, the need for relay optics is eliminated. Since the whole system is composed of just a screen and a PicoP, it is much more compacter than a virtual image HUD system. With direct projection HUD, the driver can get in the car, put the projector on the dashboard, project the content from a cell-phone running an HUD application onto the see-through screen, and start using the HUD in his/her car. The screen can be installed into any car with minor modifications, so a pico-projector and a see-through screen make an aftermarket HUD kit for cars.

This paper focuses on the design and properties of the see-through screen. Section 2 describes the geometry in which the screen is used and explains the design and structure of the screen. Section 3 presents simulations and experimental results. Concluding remarks are in Section 4.

*mhedili@ku.edu.tr; phone+90-212-338-1474; fax +90-212-338-1548; mems.ku.edu.tr

Micro-Optics 2012, edited by Hugo Thienpont, Jürgen Mohr, Hans Zappe, Hirochika Nakajima, Proc. of SPIE Vol. 8428, 84280X · @ 2012 SPIE · CCC code: 0277-786X/12/\$18 · doi: 10.1117/12.923556 


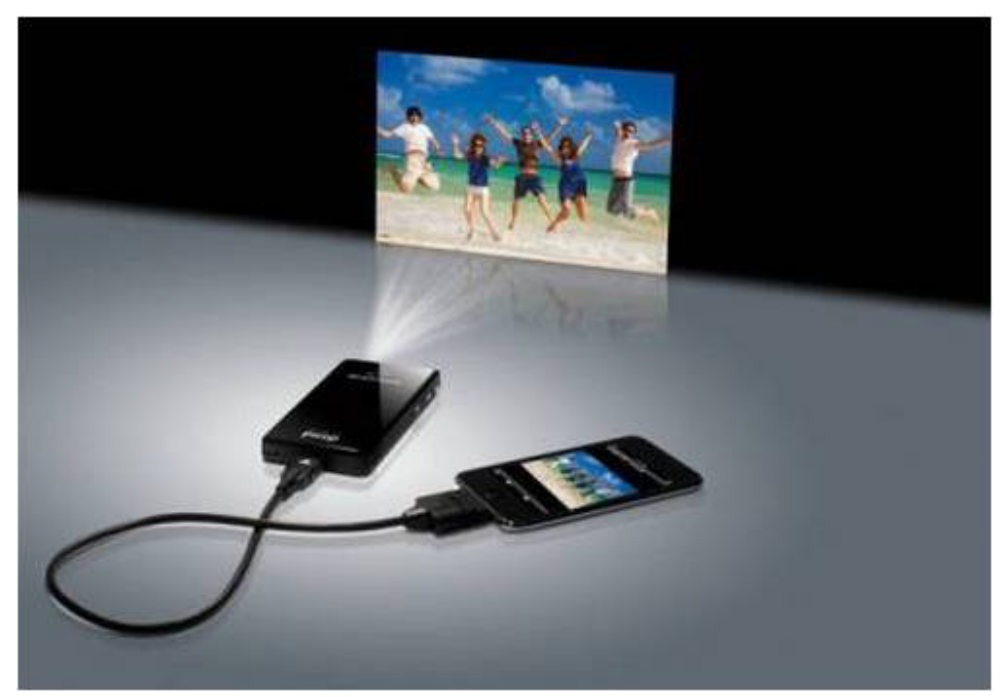

Figure 1. Using mobile pico-projectors, such as Microvision's PicoP, compact HUD systems can be designed. The driver can get in the car and place the PicoP on the dashboard and start operating the HUD system.

\section{DIRECT PROJECTION HUD SYSTEM}

\subsection{The system geometry}

The HUD system is composed of a pico projector on the dashboard and a see-through screen mounted on the dashboard, as illustrated in Fig.2. The current prototype screens measure 175x87.5mm and a typical distance to the picoprojector is about $200 \mathrm{~mm}$. The height of the driver's eyes are assumed to be $250 \mathrm{~mm}$ above the dashboard and about $1000 \mathrm{~mm}$ away from the screen. The driver has a visual field of view of approximately $10^{\circ} \times 5^{\circ}$, slightly bigger than commercially available HUDs today. The goal of the screen is to create a viewing window around the driver's eyes that is large enough for comfortable operation but as small as possible to maximize the image brightness. The screen is a partial reflector so the center of the viewing cone follows the law of reflection (angle of incidence $=$ angle of reflection). The screen and the projector can be positioned wherever they fit naturally in a car, and the screen can be tilted to steer the light to the driver's eyes.

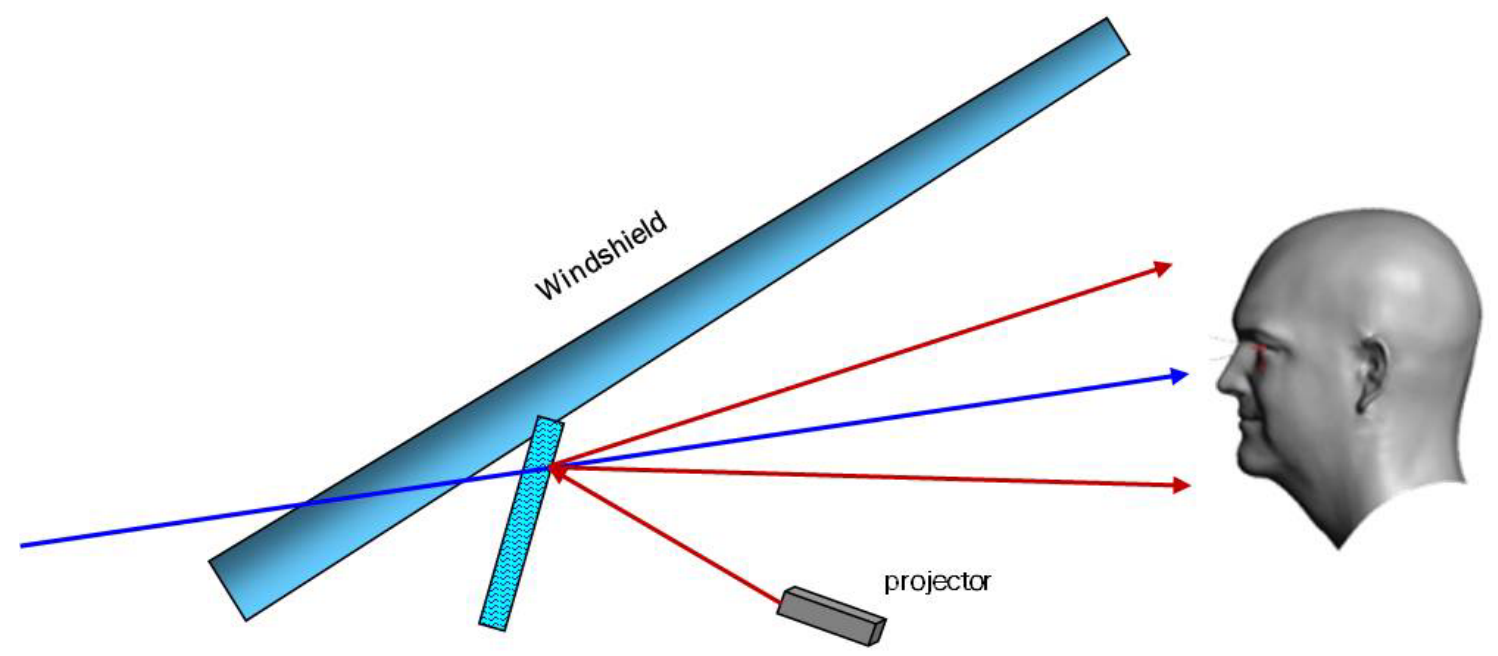

Figure 2. The screen is in front of the windshield, illuminated by the Picop on the dashboard. The screen does not distort the transmitted light but expands the reflected light to form the viewing window. 


\subsection{The structure of the screen}

A partially-reflective surface-relief diffusing screen structure is sandwiched between index-matched layers of glass and epoxy, as illustrated in Fig.3. As a result, the transmitted light sees a constant index of refraction between two planar interfaces, whereas the reflected light is expanded by the microlenses to form the viewing window. Due to the uniform index of refraction between the outer surfaces of the screen, the transmitted light experiences no refraction, and the see-through scene is therefore undistorted. It's like looking through a window. There is some reflection from the partially-reflective coating on the surface of the diffusing screen structure, but to the transmitted light, this is just a small loss in intensity. The reflected light, on the other hand, reflects back towards the driver from the microstructured, surface-relief partial reflector and appears as an image on the screen to the observer.

We chose a microlens array (MLA) for our screen structure as discussed below in detail. The microfabricated MLA mold is filled with a UV-curable epoxy and hardened by UV light exposure. This creates a microlens array on the first glass substrate. The microlenses are coated with a partially reflective coating. The coating properties can be optimized for the particular application. Once the coating has been applied to the microlens array, a second cover plate is added using an index-matched adhesive and cured with UV light once more. As the whole structure is index matched, the transmitted light is not affected by the microlenses, thus the screen has very good transparency, making it almost indistinguishable from the windshield.

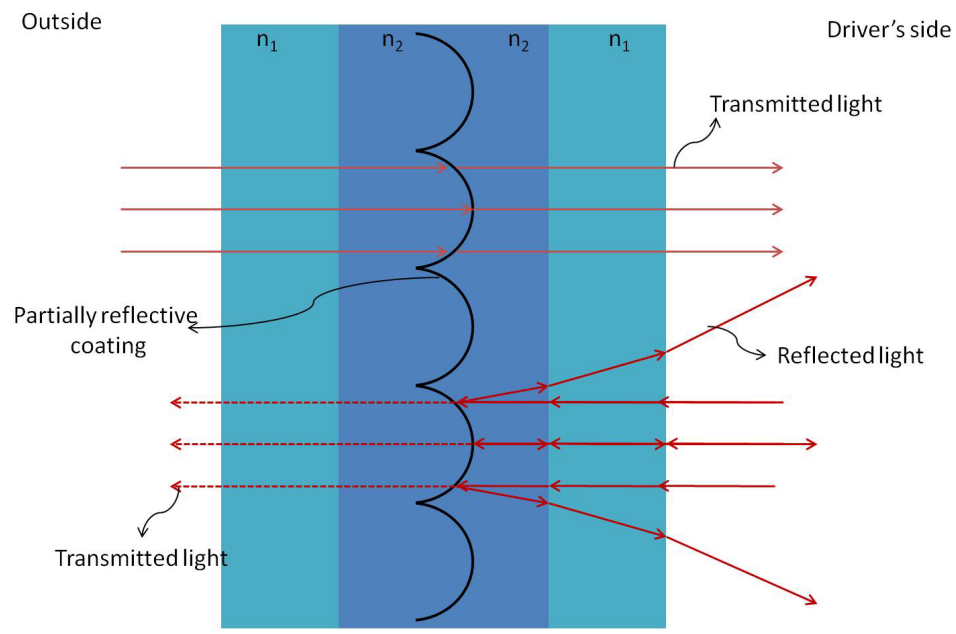

Figure 3. The screen in detail: the microlens array is sandwiched between index matching layers. The microlens array is coated with a partial reflector so that the reflected light is expanded to form the viewing window. Microlenses do not affect the transmitted light since the whole structure is index matched.

The lens pitch $(\Lambda)$ and wavelength $(\lambda)$ determines the fringe spacing $(\theta)$ according to Eq. $1^{3}$. There should be at least one diffraction order per wavelength entering the eye, so that the driver sees that color component anywhere in the viewing window. If we assume that an eye pupil is about $3-7 \mathrm{~mm}$ in diameter, the diffraction order separation should be at most $3 \mathrm{~mm}$. Since the distance between the screen and the driver is assumed to be $1000 \mathrm{~mm}$, the angular separation between the diffraction orders should be at most $0.003 \mathrm{rad}$. As the longest laser wavelength in PicoP is $635 \mathrm{~nm}$, the MLA pitch can be calculated to be about $300 \mathrm{um}$. With the calculated MLA pitch, the radius of curvature is optimized through simulations yield the desired size for the full viewing window.

$$
\sin (\theta)=\frac{\lambda}{\Lambda}
$$

We tried two different reflective coatings on the MLA, one of them is a thin metal coating and the other one is a broadband dielectric coating. With the thin metal coating, the sum of transmittance, reflectance and absorbance is equal to the incident power. With dielectric coatings, absorbance is almost zero, so the transparency of the screen is superior to the metal-coated ones. The thin metal coating is easy to apply and has good reflectance but it has about $40 \%$ absorbance. As a result the screen looks dimmer than the windshield, thus we prefer the broadband dielectric coating for the seethough screens. The outside surfaces of the cover glasses are coated with an anti-reflection coating to minimize extraneous reflections. 
We chose an MLA as the diffuser in the screen as it offers easily controllable viewing windows parameters like size and shape, has very good color balance, and no speckle making it suitable for color laser projection displays. It turns out that MLA-based exit pupil expanders are a good choice for polychromatic displays because, although the diffraction order spacing is different for different wavelengths, the viewing window sizes are determined by the microlens curvature and are the same for all colors ${ }^{4}$. As a result, the overlapping viewing windows offer a very good color balance throughout the viewing window. The size of the viewing windows can be controlled by the pitch and the radius of the curvature of the microlenses whereas the shape of the viewing windows can be controlled by the shape of the aperture of the microlenses. And since the surface of the MLA is engineered with good optical quality, random variations that would create speckle are not observed.

The see-through screen structure is not limited to using MLAs. The see-through screen concept works with any surface-relief microstructured element in place of the microlenses. For example a random diffuser or a diffractive optical element could be used instead. A random diffuser can work well with incoherent light but gives a less controlled eyebox than an MLA. If coherent light is used however, the random surface profile results in undesirable speckle due to the random interference patterns generated. A diffractive optical element such as a binary diffraction grating could also be used as an exit pupil expander ${ }^{5}$. In this case, the size of the viewing window is determined by diffraction. For different wavelengths the number of diffraction orders is the same but the viewing window sizes are different. As a result the viewing windows corresponding to different colors do not overlap very well, and the color balance degrades at the edges of the viewing window 5 .

\section{SIMULATIONS AND EXPERIMENTAL RESULTS}

The embedded MLA screen has been modeled and simulated using Zemax in the non-sequential mode. A $635 \mathrm{~nm}$ collimated Gaussian beam was used for illuminating the screen. 50 sample points, each at a different projection angle onto the screen, were modeled in the simulation: 5 equidistant rows with 10 equidistant sample points on each row. The model represents using a scanned laser projector to illuminate the MLA screen (300um MLA spacing with hexagonal arrangement of the microlenses). The range of projection angles covered 46 degrees horizontally and 25 degrees vertically. Each incident angle results in a hexagon-shaped viewing window at the driver's position. The viewing windows shift laterally as the scan angle increases. The sum of viewing windows in all their shifted positions for the 50 beams that were modeled can be seen in Fig.4. The red region in the center of the figure shows where all of the individual viewing windows overlap, that is, the useable full viewing window where every point on the screen can be seen.
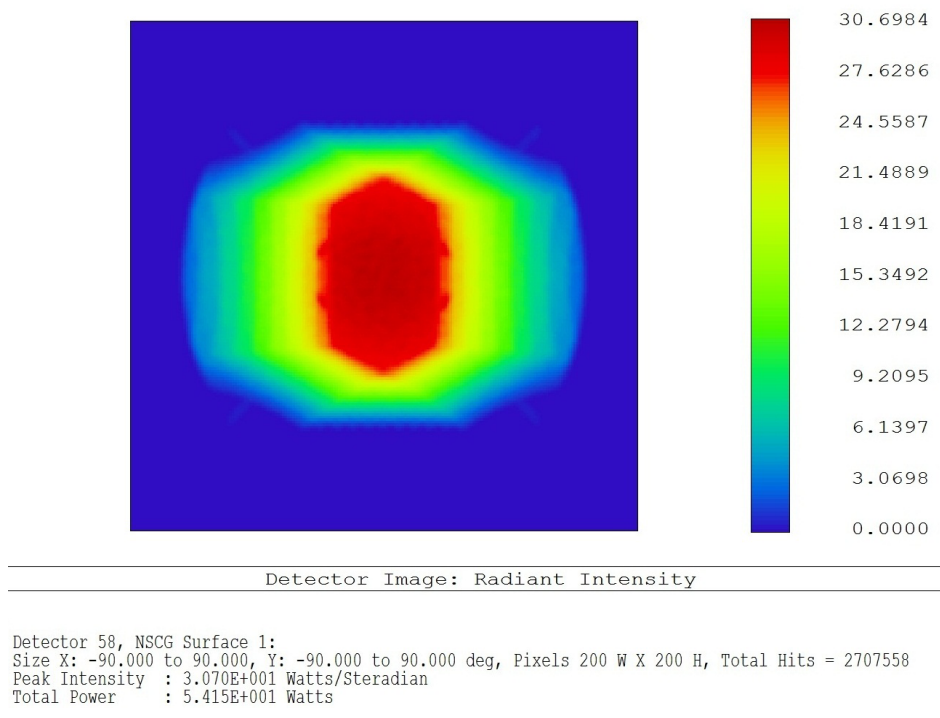

Figure 4. False color detector image of the viewing windows for 50 sample points on the screen. The red region shows the overlapping region, from where the entire screen can be seen. 
The width of the full viewing window is $\pm 18^{\circ}$ as seen in Fig. 5 , which corresponds to a width of $65 \mathrm{~cm}$ at the driver's position. The simulation results match well with screen measurements where the full viewing window was measured to be approximately $\pm 20^{\circ}$. The horizontal cross-section is quite uniform, which is more important than the vertical cross-section uniformity due to the horizontal alignment of the eyes. The vertical cross-section is about $\pm 36^{\circ}$ as seen in Fig. 6, which defines the range of driver's heights that the system can accommodate.

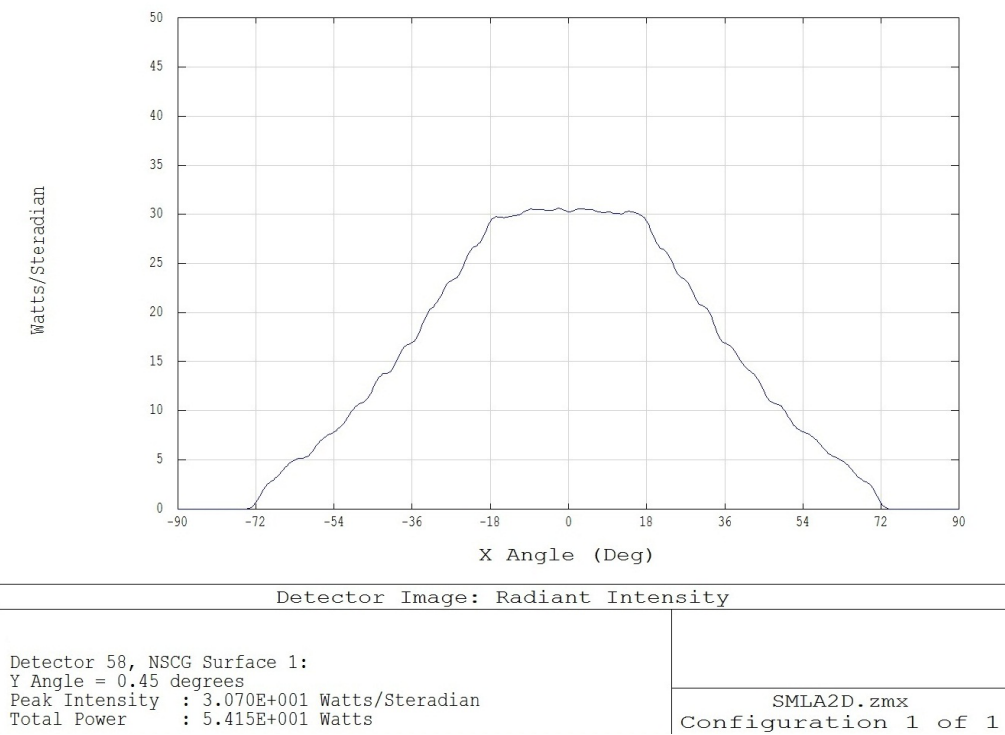

Figure 5. The horizontal cross-section of the sum of the viewing windows in Fig.4. The flat region is the cone where the entire screen is visible to the driver. $\pm 18^{\circ}$ corresponds to a width of $65 \mathrm{~cm}$ at the driver's position, which provides a comfortable operating region for the driver.

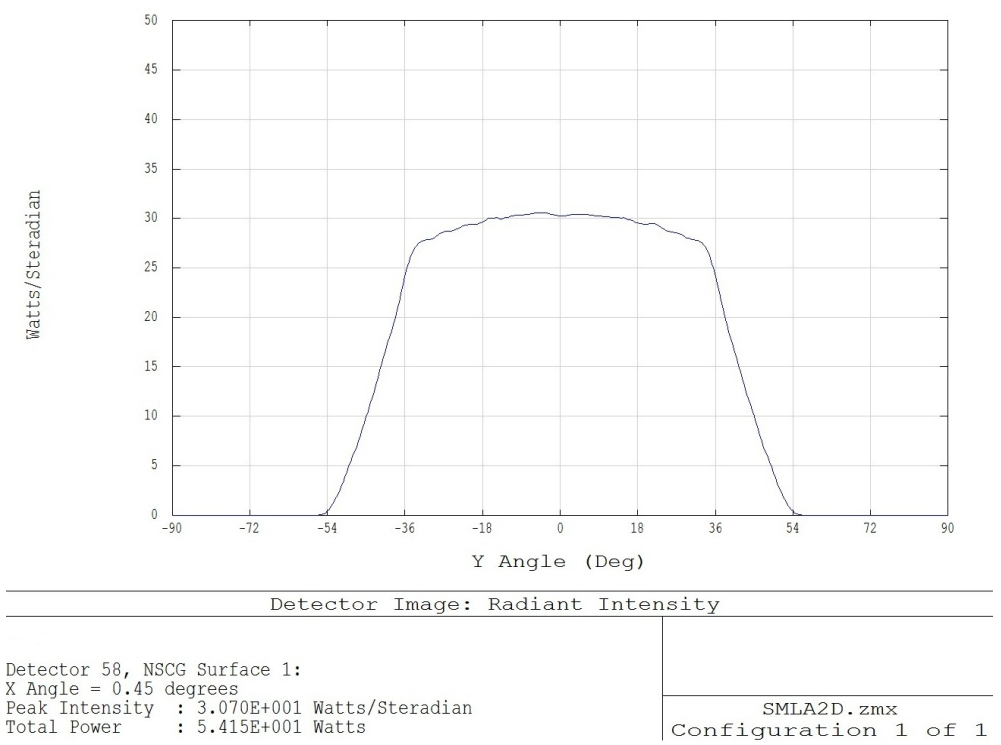

Figure 6. The vertical cross-section of the full viewing window in Fig.4. It supports a wide range of driver's heights due to the wide spread of the reflected light in the vertical direction.

The screen was fabricated as described in section 2.2, by Microvision Inc. We tested the screen in a real car, on a sunny summer day. The screen used a broadband dielectric coating with about $15 \%$ reflectance and it is indistinguishable from the windshield as seen in Fig.7. Image brightness was useable but still needs improvement for the brightest driving conditions. 


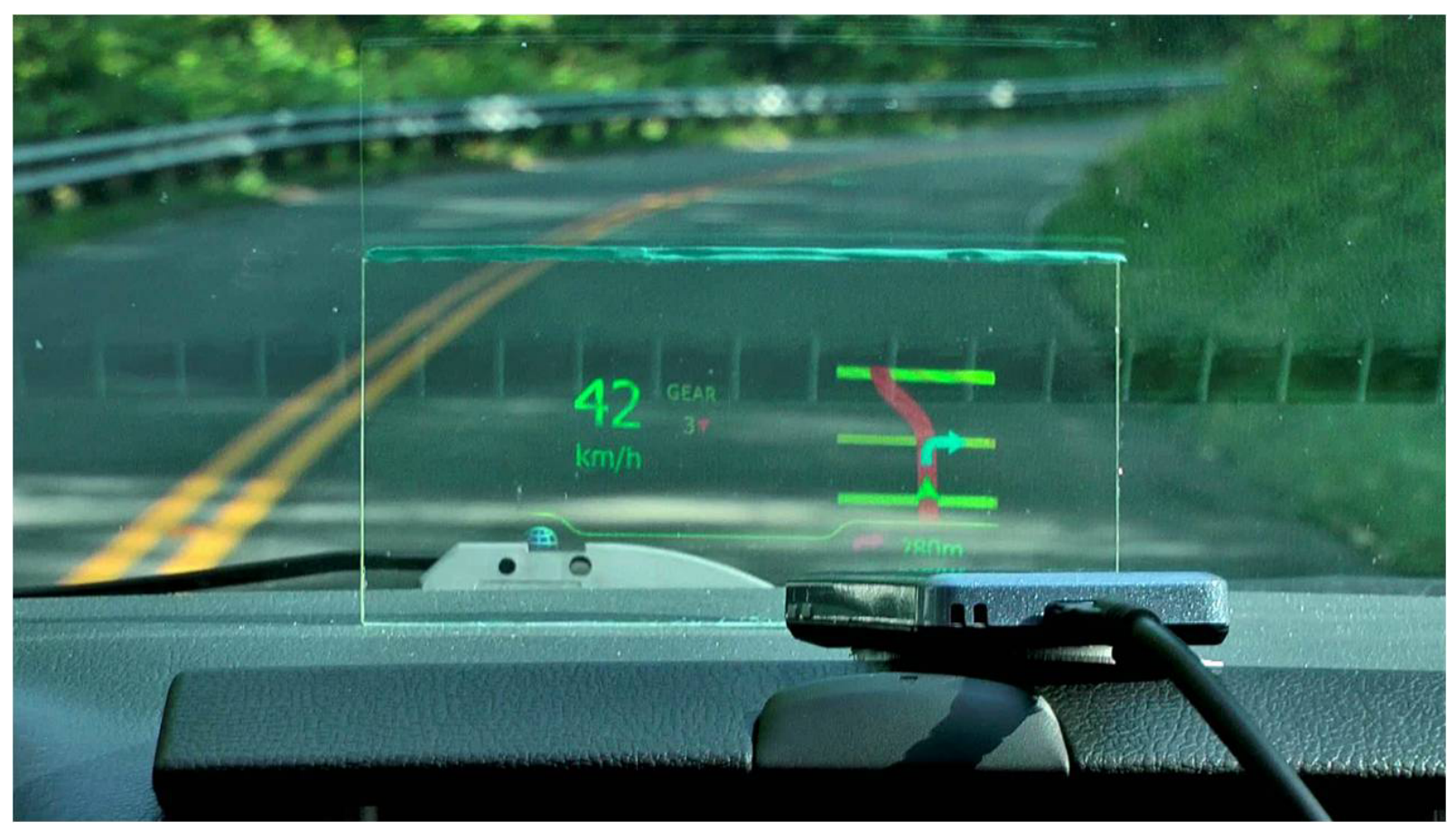

Figure 7. The system in operation in a car. Note that the screen transparency is very good and the image is bright.

\section{CONCLUSION}

We demonstrated that an MLA sandwiched between index matched layers can be used as a see-though screen for automotive HUD applications. The MLA creates a bright image with no speckle and good color balance. The wide viewing window of the screen provides a comfortable operating region for the driver. First prototypes were fabricated with two different semi-reflective coatings; a broadband dielectric and a thin metal coating. The combination of excellent transparency and a bright reflected image are quite promising for applications such as direct-projection HUD as well as other applications where it is desired to overlay information on a real-world scene. The basic structure of the screen is quite simple - a partially reflective microstructure sandwiched between two index-matched cover plates. The cover plates need not be made from glass. Plastic or other transparent materials would work as well. We believe that such a structure can be produced in large areas at a relatively low cost.

\section{REFERENCES}

[1] Liu, Y.-C. and Wen, M.-H., "Comparison of head-up display (HUD) vs. head-down display (HDD): Driving performance of commercial vehicle operators in Taiwan", Int. J. Human-Comput. Studies, vol. 61, p.679, (2004).

[2] Freeman, M. O., "MEMS scanned laser head-up display", Proc. SPIE 7930-13, Photonics West, (2011)

[3] Goodman, J.W., [Introduction to Fourier Optics, $2^{\text {nd }}$ edition], McGraw-Hill Book Co., New York, 207, (1996)

[4] Urey, H. and Powell, K. D., "Microlens-array-based exit-pupil expander for full-color displays", Appl. Opt., vol. 44, pp.4930 - 4936, (2005).

[5] Urey, H., "Diffractive exit-pupil expander for display applications," Appl. Opt. 40, 5840-5851 (2001). 